\title{
Estimates for Solutions of Semilinear Elliptic Equation in Two Dimensions
}

\author{
A and Meftah MT
}

Department of Mathematics, Faculty of Mathematics and Science, Kasdi Merbah University, Algeria

\begin{abstract}
In this paper we study a family of nonlinear Elliptic problems in two dimensions, we give some estimates for the solutions of this problem, and we decompose it on two problems, the first is the Poisson's equation and the second is the Liouville equation.
\end{abstract}

Keywords: Liouville equation; Nonlinear problem; Elliptic equation

\section{Introduction}

In this paper we study the problem

$$
\left\{\begin{array}{lr}
-\Delta u=\lambda e^{u}+f(\mathrm{x}) & \text { in } \Omega \subset \mathbb{R}^{2} \\
u=g & \text { on } \partial \Omega
\end{array}\right.
$$

Where $\Omega$ is a bounded domain, $\lambda$ is a positive parameter, $g \in L^{\infty}(\partial \Omega)$ and $f \in L^{q}(\Omega)$ for some $\mathrm{q}>1$.

Equation of Liouville-type is used in this study, it has the form:

$\left\{\begin{array}{lr}-\Delta u=v(\mathrm{x}) \mathrm{e}^{\mathrm{u}}+f(\mathrm{x}) & \text { in } \Omega \subset \mathbb{R}^{2} \\ u=0 & \text { on } \partial \Omega\end{array}\right.$

\section{$v(\mathrm{x}) \in \mathrm{L}^{\mathrm{q}}(\Omega)$ For some $\mathrm{q}>1$.}

This related equation has been received much attention in the recent years. On the one hand, this is due to the wide range of application of this equation: it used in astrophysics [1] and combution theory [2], it is also related to the prescribed Gaussian curvature problem in Riemannian geometry [3], to the mean _led limit of vortices in Euler ows [4], to onsager's formulation in statistical mechanics [5], to the Keller-Siegel system of chemotaxis [6], to the Chern-Simon-Higgs gauge theory $[7,8]$, and it has many other physical applications. On the other hand, Liouville equation is mathematically appealing since it has an interesting solution structure [9-16].

\section{Preliminaries}

Assume $\Omega \subset \mathbb{R}^{2}$ is a bounded domain and let $\mathrm{u}$ be a solution of

$\left\{\begin{array}{c}-\Delta u=f(\mathrm{x}) \text { in } \Omega \subset \mathbb{R}^{2} \\ u=g \quad \text { on } \partial \Omega\end{array}\right.$

With $f \in L^{1}(\Omega)$. set $\|\mathrm{f}\| 1=\int_{\Omega} \| f(\mathrm{x}) \mid \mathrm{dx}$

Theorem 2.1 For every $\delta \in(0,4 \pi)$ we have

$\int_{\Omega} \exp \left[\frac{(4 \pi-\delta)|\mathrm{u}(\mathrm{x})|}{\|f\|_{1}}\right] d x \leq \frac{4 \pi^{2}}{\delta}(\operatorname{diam} \Omega)^{2}$

Proof: Let $R=\frac{1}{2} \operatorname{diam} \Omega$ so that $\Omega \subset B_{R}$ for some ball of radius R.

Extend $\mathrm{f}$ to be zero outside $\Omega$ and set, for $x \in \mathbb{R}^{2}$

$\bar{u}(\mathrm{x}))=\frac{1}{2 \pi} \int_{B_{R}} \log \left(\frac{2 R}{|\mathrm{x}-\mathrm{y}|}\right)|\mathrm{f}(\mathrm{y})| \mathrm{dy}$

so that
$-\Delta \bar{u}(\mathrm{x})=|\mathrm{f}|$, on $\mathbb{R}^{2}$

note that $\bar{u}(\mathrm{x}) \geq 0$ for $\mathrm{x} \in B_{R}$ since $\frac{2 R}{|\mathrm{x}-\mathrm{y}|} \geq 1 \forall x, y \in B_{R}$ it follows

from the maximum principle that $|\mathrm{u}| \leq \overline{\mathrm{u}}$ on $\Omega$ and thus

$\int_{\Omega} \exp \left[\frac{(4 \pi-\delta)|\mathrm{u}(\mathrm{x})|}{\|\mathrm{f}\|_{1}}\right] d x \leq \int_{B_{R}} \exp \left[\frac{(4 \pi-\delta) \overline{\mathrm{u}}(\mathrm{x})}{\|\mathrm{f}\|_{1}}\right] \mathrm{dx}$

using Jensen in quality

$F\left(\int w(\mathrm{y}) \varphi(\mathrm{y}) \mathrm{dy}\right) \leq \int w(\mathrm{y}) F(\varphi(\mathrm{y})) \mathrm{dy}$

With $F(\mathrm{t})=\operatorname{expt}, \mathrm{w}(\mathrm{y})=\frac{|\mathrm{f}(\mathrm{y})|}{\|\mathrm{f}\|_{1}}$ and $\varphi(\mathrm{y})=\frac{(4 \pi-\delta)}{2 \pi} \log \frac{2 R}{|\mathrm{x}-\mathrm{y}|}$

$$
\begin{aligned}
& \int_{B_{R}} \exp \left[\frac{(4 \pi-\delta) \overline{\mathrm{u}}(\mathrm{x})}{\|f\|_{1}}\right] d x \leq \int_{B_{R}} d x \int_{B_{R}}\left(\frac{2 R}{|\mathrm{x}-\mathrm{y}|}\right)^{2-\frac{\delta}{2 \pi}} \frac{|\mathrm{f}(\mathrm{y})|}{\|\mathrm{f}\|_{1}} d y \\
& \int_{B_{R}} \exp \left[\frac{(4 \pi-\delta) \overline{\mathrm{u}}(\mathrm{x})}{\|f\|_{1}}\right] d x \leq \int_{B_{R}} \frac{|\mathrm{f}(\mathrm{y})|}{\|\mathrm{f}\|_{1}}\left[\int_{B_{R}}\left(\frac{2 R}{|\mathrm{x}-\mathrm{y}|}\right)^{2-\frac{\delta}{2 \pi}} d x\right] d y
\end{aligned}
$$

but, for $y \in B_{R}$ we have

$$
\int_{B_{R}}\left(\frac{2 R}{|\mathrm{x}-\mathrm{y}|}\right)^{2-\frac{\delta}{2 \pi}} d x \leq \int_{B_{R}}\left(\frac{2 R}{|\mathrm{x}|}\right)^{2-\frac{\delta}{2 \pi}} d x=\frac{4 \pi^{2}}{\delta}(\operatorname{diam} \Omega)^{2}
$$

and the estimate (3) follows.

Corollary 2.2 Let $\mathrm{u}$ be a solution of (2) with $f \in L^{1}(\Omega)$ Then for every constant $\mathrm{k}>0$

$$
e^{k|\mathrm{u}|} \in L^{1}(\Omega)
$$

Proof: Let $0<\varepsilon<\frac{1}{k} \quad$ We may split $\mathrm{f}$ as $\mathrm{f}=\mathrm{f} 1+\mathrm{f} 2$ with

${ }^{*}$ Corresponding author: Hichar S, Department of Mathematics, Faculty of Mathematics and Science, Kasdi Merbah University, P.O.Box 9004 Algeria, Tel: 331456848; E-mail: salihasaid70@gmail.com

Received August 06, 2014; Accepted September 25, 2014; Published September 30,2014

Citation: Hichar S, Guerfi A, Meftah MT (2014) Estimates for Solutions of Semilinear Elliptic Equation in Two Dimensions. J Appl Computat Math 3: 186. doi:10.4172/2168-9679.1000186

Copyright: ( 2014 Hichar S, et al. This is an open-access article distributed under the terms of the Creative Commons Attribution License, which permits unrestricted use, distribution, and reproduction in any medium, provided the original author and source are credited. 
Citation: Hichar S, Guerfi A, Meftah MT (2014) Estimates for Solutions of Semilinear Elliptic Equation in Two Dimensions. J Appl Computat Math 3: 186. doi:10.4172/2168-9679.1000186

Page 2 of 2

$\left\|\mathrm{f}_{1}\right\|_{1}<\varepsilon$ and $f_{2} \in L^{\infty}(\Omega)$

Write $\mathrm{u}=\mathrm{u} 1+\mathrm{u} 2$ where $\mathrm{ui}$ are the solutions of

$\left\{\begin{array}{c}-\Delta u_{i}=f_{i} \text { in } \Omega \\ u_{i}=0 \quad \text { on } \partial \Omega\end{array}\right.$

choosing, for example, $\delta=(4 \pi-1)$ in Theorem (2.1) we find

$\int_{\Omega} \exp \left[\frac{\left|\mathrm{u}_{1}(\mathrm{x})\right|}{\left\|\mathrm{f}_{1}\right\|_{1}}\right]<\infty$

And thus

$\int_{\Omega} \exp \left[\mathrm{k}\left|\mathrm{u}_{1}\right|\right]<\infty$

The conclusion follows since

$|\mathrm{u}| \leq\left|\mathrm{u}_{1}\right|+\left|\mathrm{u}_{2}\right|$

And $u_{2} \in L \infty(\Omega)$

\section{Statement of the Results}

Let $\mathrm{u}$ satisfy the nonlinear equation (Liouville Equation)

$\left\{\begin{array}{c}-\Delta u=v(\mathrm{x}) \mathrm{e}^{\mathrm{u}} \quad \text { in } \Omega \\ u=0 \quad \text { on } \partial \Omega\end{array}\right.$

Where $\Omega$ is a bounded domain in $\mathbb{R}^{2}$ and $\mathrm{v}(\mathrm{x})$ a given function on $\Omega$

Corollary 3.1 suppose $\mathrm{u}$ is a solution of (4) with $v \in L^{p}(\Omega)$ and $e^{u} \in L^{p^{\prime}}(\Omega)$ for some $1<p \leq \infty$ Then $u \in L^{\infty}(\Omega)$

Proof: By corrollary (2:2), we know that $e^{k u} \in L^{1}(\Omega), \forall \mathrm{k}>0$,

i.e., $e^{u} \in L^{r}(\Omega)$

$\forall r<\infty \quad$ It follows that $v e^{u} \in L^{p-\delta}(\Omega) \forall \delta>0 \quad$ if $p<\infty$ and $v e^{u} \in L^{r}(\Omega) \forall \mathrm{r}<\infty$ if $p=\infty$. Standard elliptic estimates imply that $u \in L^{\infty}(\Omega)$

Resolution of the equation $-\Delta u=\lambda e^{u}+f(\mathrm{x})$

The Corollary (3:1) still holds for a solution $u$ of

$\left\{\begin{array}{cc}-\Delta u=\lambda e^{u}+f(\mathrm{x}) \text { in } \Omega \\ u=g & \text { on } \partial \Omega\end{array}\right.$

With $\Omega \subset \mathbb{R}^{2}$ is a bounded domain, $g \in L \infty(\partial \Omega)$ and $f \in L^{q}(\Omega)$ for some $\mathrm{q}>1$.

Let $\mathrm{w}$ be a solution of

$\left\{\begin{array}{c}-\Delta w=f(\mathrm{x}) \text { in } \Omega \\ w=g \quad \text { on } \partial \Omega\end{array}\right.$

So that $w \in L^{\infty}(\Omega)$

The function $\mathrm{k}=\mathrm{u}-\mathrm{w}$ satisfies:

$\left\{\begin{array}{cc}-\Delta k=\left(\lambda \mathrm{e}^{\mathrm{w}}\right) \mathrm{e}^{\mathrm{k}} & \text { in } \Omega \\ k=0 & \text { on } \partial \Omega\end{array}\right.$
The solution $\mathrm{k}$ is of the problem of liouville (7). Thus the solution of the problem (5) is $\mathrm{u}=\mathrm{k}+\mathrm{w}$ with $\mathrm{w}$ the solution of the problem (6) and $\mathrm{k}$ the solution of the problem (7).

\section{Remark 3.2}

Corollary (3:1) is not valid for $\mathrm{p}=1$ for that we have this example.

Example 3.3 Let $0<\mathrm{a}<1$. The function $u=-a \log \left(\log \frac{e}{r}\right)$, with $r=|\mathrm{x}|$ satisfies

$\left\{\begin{array}{clrl}-\Delta u & =v e^{u} & & \text { in } \Omega=B_{1} \\ u & =0 & & \text { on } \partial \Omega\end{array}\right.$

With

$$
v=-\frac{a}{r 2\left(\log \frac{e}{r}\right)^{2-a}}
$$

Note that $v \in L^{1}(\Omega), \mathrm{e}^{\mathrm{u}} \in L \infty(\Omega)$ and nevertheless $u \notin L^{\infty}(\Omega)$ since $u(\mathrm{x}) \rightarrow-\infty$ as $\mathrm{x} \rightarrow 0$. The same function $\mathrm{u}$ with $\mathrm{a}<0$ provides an example where $\mathrm{u}$ satisfies (8) with $v \in L^{1}(\Omega), v e^{u} \in L^{1}(\Omega)$ and nevertheless $u^{+} \notin L^{\infty}(\Omega)$ since $u(\mathrm{x}) \rightarrow+\infty$ as $\mathrm{x} \rightarrow 0$

\section{References}

1. Gelfand IM (1963) problems in the theory of quasilinear equations. Amer Math Soc Transl 29: 295-381.

2. Kazdan JL, Warner F (1975) Existence and conformal deformation of metrics with prescribed Gaussian and scalar curvatures. Ann Math 101: 317-331.

3. Chanillo S, Kiessling M (1994) symmetry of solutions of some nonlinear problems in statistical mechanics and in geometry. Comm Math Phys 160: 217238

4. Caglioti E, Lions PL, Marchioro C, Pulvirenti M (19952) A special class of stationary flows for two-dimensional Euler equations: a statistical mechanics description, I, II. Comm Math Phys 143 174: 229-260.

5. Senba T, Suzuki T (2000) structures of the solutions set from stationary system of chemotaxis. Adv Math Sci Appl 10: 191-224.

6. Chae D, Imanuvilov O (2000) existence of non-topological multivortex solutions in the relativistic self-dual Chern-Simons theory. Comm Math Phys 215: 119142.

7. Struwe M, Tarantello G (1998) on multivortex solutions in Chern-Simons gauge theory, Boll. Unione Mat. Ital. Sez B Artic Ric Mat 8: 109-121

8. Brezis H (1983) Analyse Fonctionnelle, Masson.

9. Chandrasekhar S (1957) An introduction to the study of stellar structure, Dover, New York 1957.

10. Chen CW, Li (1991) Classification of solutions of some nonlinear elliptic equations, preprint.

11. Weston VH (1978) On the asymptotic solution of a partial differential equation with an exponential nonlinearity. SIAM J Math Anal 9: 1030-1053.

12. Nagasaki K, Suzuki T (1990) analysis for two-dimensional elliptic eigenvalue problems with exponentially dominated nonlinearities. Asymptotic Anal 3: 173188

13. Gilbarg D, Trudinger NS (1983) partial differential equations of second order Springer-Verlarg

14. Li YY, Shafrir I (1994) Blow-up analysis for solutions of $-\Delta u=v e^{u}$ in dimension two, Indiana Univ. Math J 43: 1255-1270.

15. Pino MD, Kowalczyk M, Musso M (2005) Singular limits in Liouvilletype equations, Calc. Var. Partial Differential Equations 24: 47-81. 16. Liouville J (1853) Sur l'equationaux differences partielles $\frac{d^{2} \log \lambda}{d u d v} \pm \frac{\lambda}{2 a^{2}}=0, \mathrm{~J}$
Math Pures Appl 18: 71-72. 